Gynecologic and

Obstetric Investigation
Gynecol Obstet Invest 2011;72:79-84

DOI: $\underline{10.1159 / 000323672}$
Received: May 3, 2010

Accepted after revision: December 1, 2010

Published online: August 17, 2011

\title{
Voiding Dysfunction after the Tension-Free Vaginal Tape Procedure
}

\author{
Andreas Reich $^{\mathrm{a}}$ Frauke Kohorst $^{\mathrm{a}}$ Rolf Kreienberg ${ }^{\mathrm{a}}$ Felix Flock ${ }^{\mathrm{b}}$ \\ ${ }^{\mathrm{a}}$ Department of Obstetrics and Gynecology, University of Ulm, Ulm, and ${ }^{\mathrm{b}}$ Department of \\ Obstetrics and Gynecology, Clinical Centre of Memmingen, Memmingen, Germany
}

\section{Key Words}

Tension-free vaginal tape $\cdot$ Voiding dysfunction $\cdot$ Residual urine volume

\begin{abstract}
Aim: To assess the incidence of voiding dysfunction in women undergoing a tension-free vaginal tape (TVT) procedure and report our experience with nonsurgical and surgical management. Methods: Pre- and postoperative introital ultrasonographic measurement of the residual volume was performed in a prospective observational study. Patients' perception of micturition and continence status was assessed by questionnaire. Results: Of 478 women, 4 (0.8\%) suffered from micturition disturbance within the first 2 weeks. In the mean of 3 months after surgery, $7.1 \%$ of the patients had residual volume between 50 and $100 \mathrm{ml}$, and $2.6 \%$ had residual volume $>100 \mathrm{ml}$. 243 women were investigated after 39 months (range 12-74). The rate of residual volume exceeding $50 \mathrm{ml}$ decreased to $6.5 \%$ and was approximated to the preoperative period. Conclusion: Incomplete bladder emptying is a possible problem after TVT. In our cohort, $93 \%$ of the women had no voiding disturbances or marginally affected voiding after 12-74 months.
\end{abstract}

Copyright ๑ 2011 S. Karger AG, Basel
(C) 2011 S. Karger AG, Basel

0378-7346/11/0722-0079\$38.00/0

Fax +4161306 1234

E-Mail karger@karger.ch

www.karger.com
Accessible online at:

www.karger.com/goi

\section{Introduction}

Suburethral slings with tension-free vaginal tapes (TVT) have become a widespread treatment of female stress urinary incontinence (SUI) since their first description by Ulmsten et al. [1]. In the meantime, an important study is available regarding the long-term results of the TVT procedure with a follow-up time of 11 years with a subjective cure rate of $77 \%$ [2]. However, despite its popular use as the most widely used surgical procedure for SUI, TVT is associated with a significant complication rate. According to large observational studies [3-6], various complications have been described like bladder perforation (2.7-5.9\%), retropubic hematoma (2-3.4\%), erosion of the vaginal wall $(0.2-0.7 \%)$, and injury of the bowel $(0-0.04 \%)$. Furthermore, frequent problems after the TVT procedure are voiding dysfunctions, from mild changes in micturition to urine retention. We found conflicting publications about this complication and the value of different types of management [7-14]. The aim of this prospective observational study is to investigate the frequency and degree of voiding dysfunction and their appropriate management.

\section{Materials and Methods}

The TVT procedure (Gynecare Inc., Somerville, N.J., USA) was introduced in our department in August 1998. For this study, we included all 478 consecutive patients who were operated on

Dr. med. Andreas Reich

Department of Obstetrics and Gynecology

University of Ulm, Prittwitzstrasse 43

DE-89075 Ulm (Germany)

Tel.+49731 5005 8501, E-Mail andreas.reich@uniklinik-ulm.de 
between October 1998 and December 2005. All patients suffered from genuine SUI grades I-III according to the Ingelman-Sundberg scale [15]. Multichannel urodynamic studies together with assessments of residual urine volume were performed preoperatively. The surgical technique was similar to that described by Ulmsten et al. [1]. All TVT operations were performed by two surgeons (F.F. or A.R.). Each patient received prophylactic antibiotics for 3 days outside the recommendations. A catheter was introduced into the urethra, and the bladder was drained for $24 \mathrm{~h}$.

We examined and interviewed the patients after TVT implantation before discharge in the mean after 2 days and after an average of 3 months (range 2-9). Up to the time of data collection we were able to include 264 women treated between 1998 and 2002 at least 12 months after the TVT procedure (mean 39, range 12-74). The follow-up was carried out using a standardized questionnaire (ICIQ-FLUTS, long form), gynecological examination, stress test in the supine and upright position with a comfortably filled bladder and 24-hour pad test. A woman was considered objectively dry if the result of the stress test was negative and no stress incontinence episodes were reported verbally by the woman or recorded in her questionnaire and the pad test showed $<5 \mathrm{mg}$ urine loss per day (without reference in the literature). At the first follow-up before discharge we did the investigation without the pad test. Subjective continence status and micturition was determined by the subjects' responses to specific questions in a standardized questionnaire. The patients' postvoid residual bladder volume was estimated by introital ultrasound. Ultrasound imaging was used to obtain sagittal and transverse images of the largest cross sections of bladder visualized. The diameters were measured in three orthogonal directions. From the top to the bottom of the bladder $(\mathrm{Y})$ and at $90^{\circ}$ to this diameter $(\mathrm{Z})$ in the sagittal plane, and from left to right in the transverse plane $(\mathrm{X})$. The residual volume was calculated using the formula for approximation of the ellipsoid $(\mathrm{X} \times \mathrm{Y} \times \mathrm{Z} \times 0.81)$. All patients were prospectively followed. The Statistical Package for Social Sciences was used for data analysis (SPSS for Windows). The $\chi^{2}$ test was applied for the categorical variables. Probability values of $<0.05$ were considered statistically significant.

\section{Results}

We considered the clinical results from all 478 patients. $90 \%$ of the women underwent their first incontinence surgery. In $26 \%$ of the patients, the TVT procedure was combined with other pelvic surgery, in particular prolapse surgery. TVT alone was performed in most cases (71\%) under local anesthesia with intravenous analgesic sedation (remifentanil). The additional surgeries were carried out using spinal or general anesthesia. Basic characteristics are shown in table 1.

\section{Early Postoperative Voiding Dysfunction after TVT}

Within the first 2 weeks after the TVT procedure, 4 women $(0.8 \%)$ suffered from decelerated and accumulated micturition ( $>8 /$ day) with residual volume of $250-700$
Table 1. Basic characteristics of the patients $(n=478)$

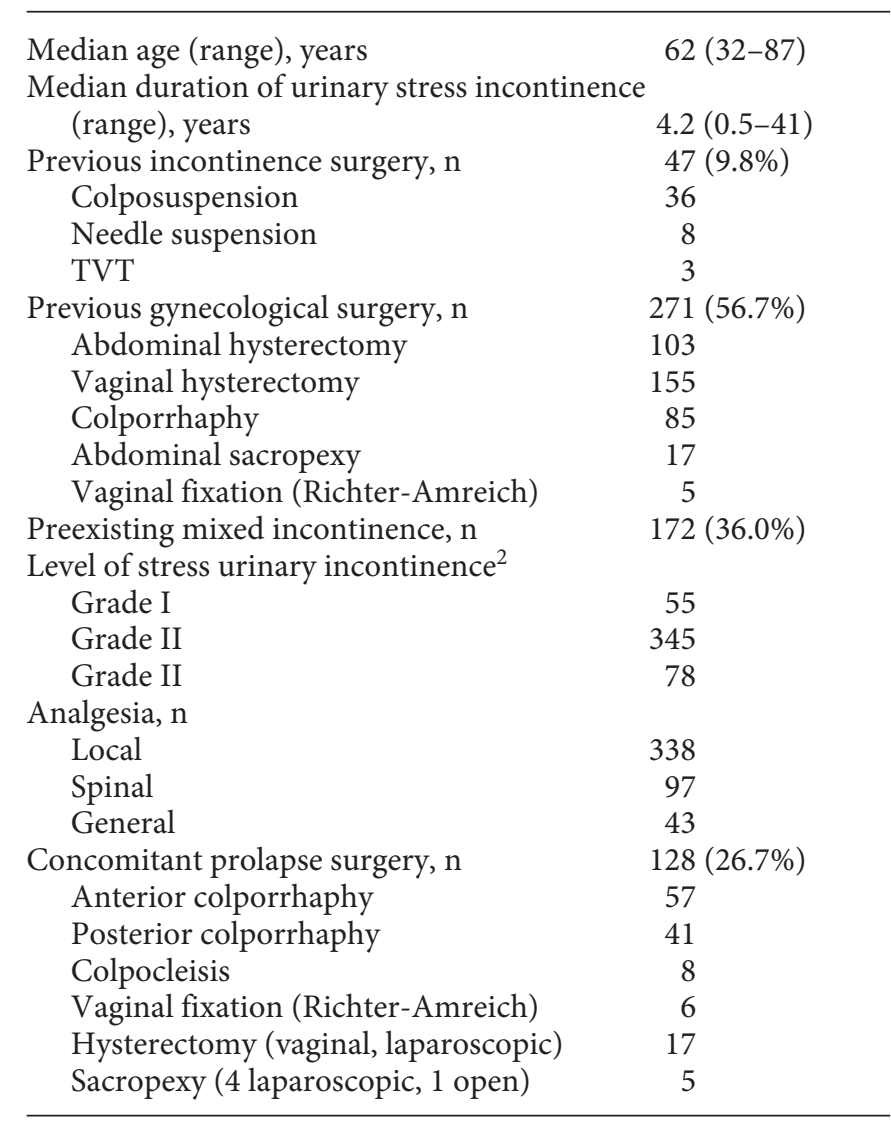

$\mathrm{ml}$. All women in our collective with residual volume $<250 \mathrm{ml}$ have no accordant symptoms. Primarily we tried to treat the symptomatic patients with repeated catheterization and administration of an $\alpha$-adrenergic receptor antagonist (phenoxybenzamine) because of the possibility of a postoperative turgor or a detrusor-sphincter imbalance. After unsuccessful conservative therapy a surgical intervention was performed after 5-14 days. In the first 2 cases we opened the vaginal incision and cut through the suburethral tape. Then we sewed in a prolene mesh $(0.8 \times 1.5 \mathrm{~cm})$ as a connecting piece. Both patients had no disturbance of micturition with irrelevant residual volumes between 0 and $40 \mathrm{ml}$ after the procedure. One patient developed SUI after 3 months. This method is awkward to perform because of the difficulty of fixing the patch to the constricted ends of the tape. Therefore, we changed our management in the next 2 cases. After opening the vaginal incision, we clamped the tape on both sides as lateral as possible, enabling us to pull the tape in the dorsal direction. In this way the suburethral part of the tape remains on the original structure. After- 
Table 2. Correlation between residual volume and subjective report of voiding dysfunctions in the mediumterm follow-up (mean 3 months)

\begin{tabular}{lrrll}
\hline \multicolumn{5}{c}{ Subjective voiding dysfunctions } \\
\cline { 2 - 5 } & unaltered & $\begin{array}{l}\text { residual volume } \\
\text { without discomfort }\end{array}$ & $\begin{array}{l}\text { residual volume with } \\
\text { moderate discomfort }\end{array}$ & $\begin{array}{l}\text { residual volume with } \\
\text { severe discomfort }\end{array}$ \\
\hline $\begin{array}{l}\text { Residual volume } \\
0-49 \mathrm{ml}\end{array}$ & $267(64.8 \%)$ & $130(31.5 \%)$ & $11(2.7 \%)$ & $4(1 \%)$ \\
$50-100 \mathrm{ml}$ & $19(57.6 \%)$ & $8(24.2 \%)$ & $4(12.1 \%)$ & $2(6.1 \%)$ \\
$>100 \mathrm{ml}$ & $5(41.6 \%)$ & $2(16.7 \%)$ & $3(25 \%)$ & $2(16.7 \%)$ \\
\hline
\end{tabular}

wards neither patient showed further obstructive symptoms or even retention. These patients were still continent after 3 and 4 months.

In addition, we saw a moderate residual volume between 50 and $150 \mathrm{ml}$ in a further $80(17 \%)$ of the $478 \mathrm{pa}-$ tients. These women were satisfied with the micturition, thus no surgical intervention was indicated.

\section{Medium-Term Voiding Dysfunction (Mean 3 Months) after TVT}

We performed a follow-up examination in 463 women between 2 and 9 months (mean 3) after the TVT operation. This means a collection of data in $97 \%$. Three months after surgery, $88.8 \%$ of the women were objectively cured of SUI. In $9.7 \%$ we found an improvement, and in $1.5 \%$ the SUI persisted. A total of $91.6 \%$ of the patients evaluated the result of the surgery as 'good' or 'very good', $6.8 \%$ considered the result 'moderate', and $1.6 \%$ 'unsatisfactory'. 126 patients (27.2\%) suffered from urge incontinence (preoperatively 165 patients, 35.6\%).

Subjectively, $63.7 \%$ of the patients reported that the voiding phase was unaltered or even improved. According to all accounts, $30.6 \%$ of the patients had a prolongation of the voiding phase without disturbance of the quality of life. Otherwise, 3.9\% of the women reported that voiding difficulties were causing them moderate discomfort and $1.8 \%$ severe discomfort. Patients with more residual volume had also more subjective voiding dysfunctions as expected (table 2).

In the medium-term follow-up after surgery, 7.1\% ( $\mathrm{n}=$ 33 ) of the patients had residual volume between 50 and $100 \mathrm{ml}$ (median 60). Twelve patients (2.6\%) had residual volume exceeding $100 \mathrm{ml}$. Only 14 (31.1\%) of these 45 patients with residual volume had residual volume in the early postoperatively period. We found no relation be- tween voiding dysfunction in women without concomitant anterior colporrhaphy or concomitant anterior colporrhaphy (10.1 vs. $7 \%)$.

In 2 patients $(0.4 \%)$ we performed a tape transection 5 and 6 months after TVT implantation because of refractory postoperative voiding dysfunction with incomplete bladder emptying with residual volume of 80 and $350 \mathrm{ml}$ respectively. First, we localized the sling with a calibration of the urethra by a bougie (thin cylinder of metal with a rounded cone end). During extraction of the bougie from the urethra it is easy to feel a 'step' at the position of the tape. We performed a small incision of the anterior vaginal wall. We favored a unilateral transaction (5-8 $\mathrm{mm}$ paraurethral) because the distance to the urethra by the lateral dissection is greater than when cutting directly under the urethra in the medial direction. Furthermore, after lateral transection the urethra is continually supported by a part of the tape. After undermining the tape, we intersected the complete tape and closed the incision with suture. Both patients were discharged the next day. Eight weeks later both women were satisfied with the result and had no residual volume; however, both showed recurrence of SUI.

Overall, during the first year after surgery, surgical intervention was necessary in $1.3 \%$ of 463 patients after the TVT procedure due to voiding dysfunction.

\section{Long-Term Voiding Dysfunction (Mean 39 Months,}

Range 12-74 Months) after TVT

To assess the long-term changes of micturition, we included 264 women in the study. In the meantime, 11 women had died, 3 were in need of care, and 7 could not be reached by telephone or post. Therefore, we examined 243 women more than 1 year after surgery. 
Table 3. Literature regarding surgical intervention because of voiding dysfunction after TVT

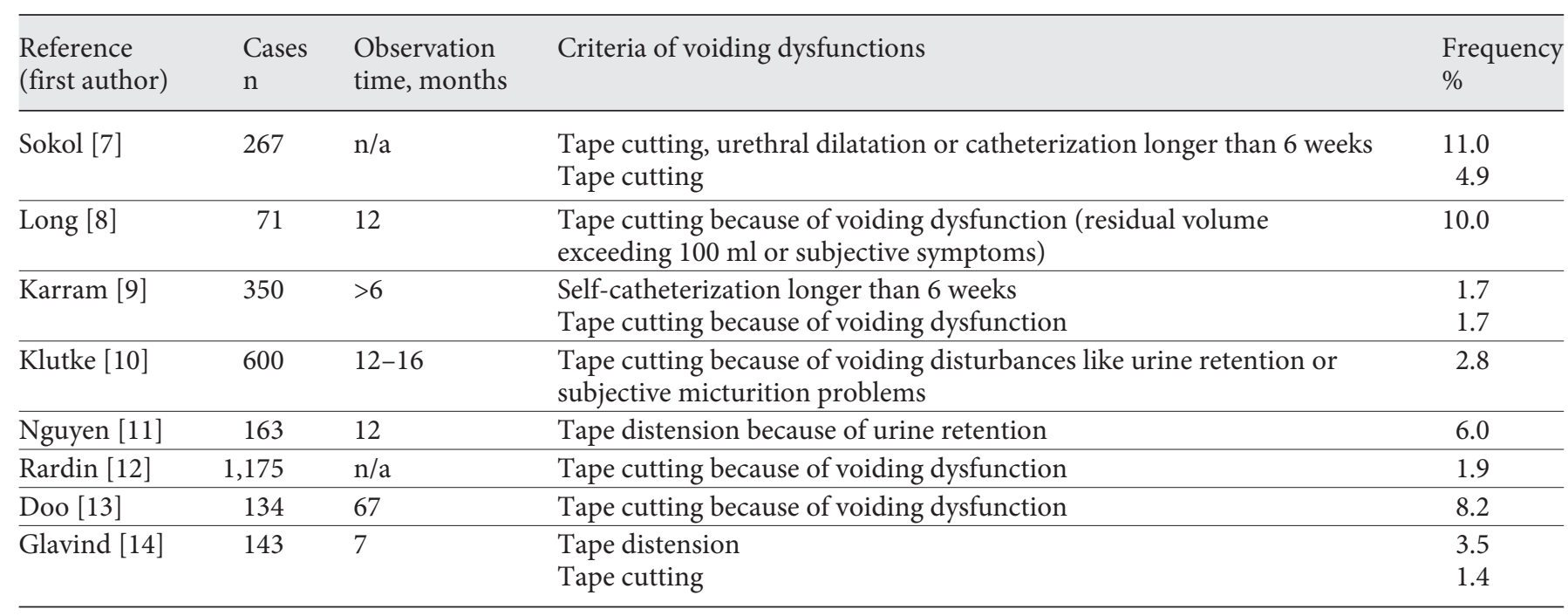

$\mathrm{n} / \mathrm{a}=$ Not applicable.

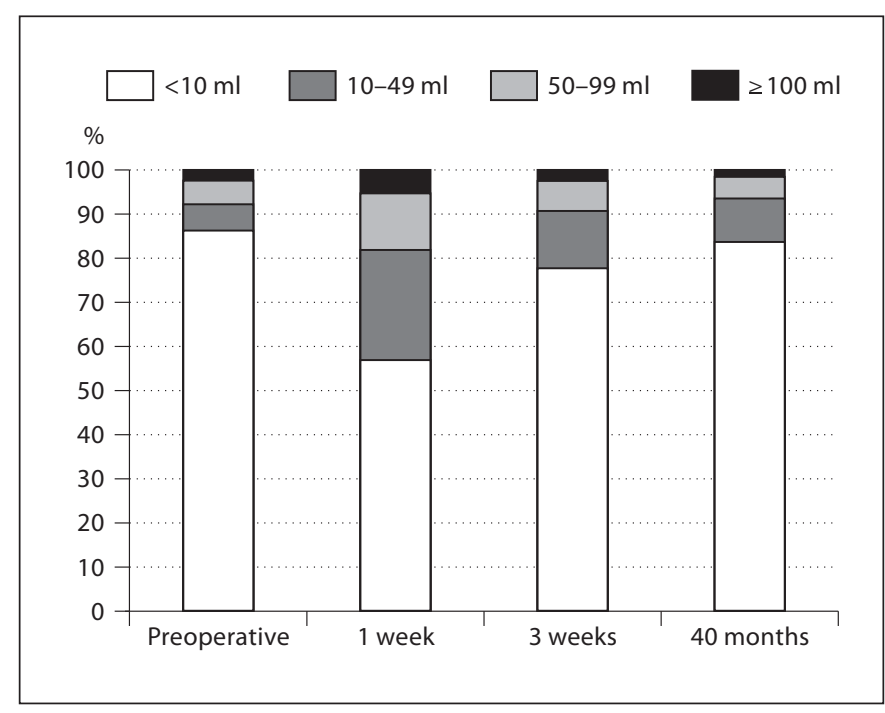

Fig. 1. Frequency of residual volume in the long-term follow-up after the TVT procedure among 243 women.

SUI was cured objectively in $80.7 \%$ of the women. Incontinence was improved in $5.2 \%$ and there was persistent or worsened SUI in only $4.2 \%$. In comparison with the medium-term follow-up the cure rate is lower $\left(\chi^{2}\right.$ test, $\mathrm{p}=0.009)$. Overall, $81.8 \%$ of the patients were satisfied with the results of the surgery ('good' or 'very good') and $4 \%$ were 'unsatisfied.' In contrast to the medium-term results this shows a significant difference $\left(\chi^{2}\right.$ test, $\left.\mathrm{p}<0.001\right)$.
In the long-term follow-up, $6.5 \%$ of the women had a residual volume exceeding $50 \mathrm{ml}$ and $1.7 \%$ exceeding 100 $\mathrm{ml}$. Subjectively, 13 women (5.3\%) described voiding disturbances as 'disturbing' and 3 women (1.2\%) as 'very disturbing.' Only 4 of these 16 women with subjectively disturbed micturition had residual volume between 30 and $70 \mathrm{ml}$. None had recurrent bladder infections; thus we refrained from cutting the tape. $93 \%$ of the women after the TVT procedure showed no disturbance of or marginally affected voiding. We found an approximation of the residual volumes to the preoperative volumes over time. The rate of relevant residual volume exceeding $50 \mathrm{ml}$ in the long-term follow-up was not higher than preoperatively. The results concerning residual volume in the long-term follow-up are shown in figure 1.

\section{Discussion}

Postoperative voiding dysfunctions are a typical complication of all incontinence surgeries. Also after tensionfree suburethral tape procedures, voiding dysfunctions are relatively common and the most frequent postoperative problem. Typical symptoms are micturition with abdominal press, weak urinary stream, micturition in small quantities and urgency or the sensation of residual volume.

In the literature there exists a wide range of the frequency of micturition disturbances from 1 to $26 \%[9,16-$ 
18] because of different definitions. In one study [19], the rate of subjective voiding dysfunction was even $60 \%$. The different boundary values of residual volume are not helpful in the clinical practice. Rather the symptomatology is the basis of decision. A high-grade voiding dysfunction is usually the cause of a surgical intervention. It is to be assumed that the frequency of such procedures is better comparable than imprecise definitions mentioned above. According to large observational studies $[3,4-6]$ the rate of voiding dysfunction with the need of surgical intervention is $0.2-1.6 \%$. The studies with the focus on surgical management of voiding dysfunction determined a proportion of surgical intervention because of urinary retention from 1.7 to $11 \%$ (table 3). Various factors were shown to be associated with postoperative voiding difficulties, including the age of the patient [20-22], abnormal preoperative uroflow pattern $[20,21,23,24]$, general anesthesia [23], previous incontinence surgery [21], an inexperienced surgeon [8], recurrent surgery [19], concurrent vault suspension surgery [7], and postoperative urinary tract infection [7]. Other authors do not corroborate these risk factors $[10,25]$. The most feasible risk factor, in our opinion, is the disrespect of the tension-free application of the sling.

There are different suggestions to manage an overcorrection. In the starting years of TVT, several colleagues attempted to release the sling with a bougie intraurethrally $[9,26]$. The benefit of this method was never examined in studies, and secondary penetrations of the sling into the urethra occurred. This procedure is therefore no longer recommended. Instead it is suggested that the sling should be dissected by a vaginal incision. In the first 2 postoperative weeks the tape is normally well presentable, so that, lateral on both sides, the tape can be clamped and released with traction in the dorsal direction [11, 26]. Some weeks after TVT implantation, the tape is interspersed with scar tissue to ensure that tape distension is no longer possible. In these cases a transection of the tape central below the urethra $[10,12]$ or lateral to the urethra [8] is suggested. In our opinion, the advantage of the lateral transection is the decreased risk of a urethral lesion, and potentially a lateral transection is able to reduce the risk of a recurrence of SUI because the unaffected part of the tape under the urethra may still support the continence system. But until now it is unclear where we should cut the tape. Some authors recommend a partial resection of the tape to avoid reunification of the tape $[3,10,27]$. It has not yet been demonstrated that an additional resection of the tape is beneficial.

In the early postoperative period we performed the incision under the urethra to release the sling, and after 2

Voiding Dysfunction after the TVT Procedure weeks we cut the tape laterally. According to the results from other authors $[8,10-12]$, no patients showed any disturbances of urination after that procedure. The continence rate after surgical revision is about $70 \%[8-10,12]$.

At present there are no uniform recommendations concerning the best time to intervene in the case of voiding dysfunction after TVT. Tension-free placement of the tape may not prevent the development of postoperative voiding difficulties in all cases. Postoperative edema and reflectoric disturbances of urination are worthy of consideration. In a study by Nguyen [11], all patients received a suprapubic catheter during the TVT procedure. The patients without voiding difficulties needed $33 \mathrm{~h}$ after TVT alone and $74 \mathrm{~h}$ after TVT with concomitant prolapse surgery. Other studies showed a period of normalization with a mean of 5 days after TVT alone and 8 days after TVT with other surgeries $[7,28]$.

In spite of a tension-free application, the TVT procedure tends to result in an increased urethral resistance factor. Most of the studies with urodynamics showed a significant decrease of the flow rate months and years after TVT placement $[13,29,30]$. Only a few authors presented normal flow rates after TVT [5, 31]. Two longitudinal studies show an improvement of micturition over the years without reaching the preoperative values [13, 29]. In this respect it is possible to initially wait in the case of voiding dysfunction 6-8 weeks after the TVT procedure and to bridge the time gap with self-catheterization or a suprapubic catheter [27]. After this waiting period a revision such as previously described is necessary when there is no improvement of the voiding problems. Otherwise an early intervention is reasonable because in the first postoperative weeks it is possible to release the sling without cutting the tape. This is probably the best course of action in cases of a close-fitting sling because waiting patiently does not solve the problem. In this regard it is helpful to evaluate the tape by ultrasound. We arranged an own study to that question with introital ultrasound [32]. Regarding the distance between tape and urethra there were significant differences in patients with residual volume compared with women without residual volume. There was a clear increase of the rate of residual urine in patients with a distance between tape and urethra $<3 \mathrm{~mm}$. Thus, we suggest measuring the distance between tape and urethra by ultrasound in cases with residual volume. If the distance is $<3 \mathrm{~mm}$, it is presumable that the tape is too tight and causal for the voiding dysfunction. In these cases we recommend releasing the tape within 2 weeks after surgery to avoid cutting the tape at a later point in time.

Gynecol Obstet Invest 2011;72:79-84 
A limitation of our study is the omission of urodynamic measurements in cases of postoperative residual volume to differentiate vesical from postvesical disturbances.

In conclusion, the TVT procedure is associated in our collective with symptomatic incomplete bladder emptying in the early postoperative period in $0.8 \%$, in the me- dium-term period in $3.9 \%$ with moderate discomfort and in $1.8 \%$ with severe discomfort, and in the long-term follow-up in $5.3 \%$ in a 'disturbing' manner and in $1.2 \%$ in a 'very disturbing' manner. Refractory voiding dysfunctions with the necessity of surgical intervention is a relatively uncommon situation.

\section{References}

1 Ulmsten U, Henriksson L, Johnson P, Varhos G: An ambulatory surgical procedure under local anesthesia for treatment of female urinary incontinence. Int Urogynecol 1996;7: 81-86.

-2 Nilsson CG, Palva K, Rezapour M, Falconer $\mathrm{C}$ : Eleven years prospective follow-up of the tension-free vaginal tape procedure for treatment of stress urinary incontinence. Int Urogynecol J Pelvic Floor Dysfunct 2009; 19:1043-1047.

>3 Tamussino KF, Hanzal E, Kolle D, Ralph G, Riss PA: Tension-free vaginal tape operation: result of the Austrian Registry. Obstet Gynecol 2001;98:732-736.

4 Kuuva N, Nilsson CG: A nationwide analysis of complications associated with tensionfree vaginal tape (TVT) procedure. Acta Obstet Gynecol Scand 2002;81:72-77.

5 Meschia M, Pifarotti P, Bernasconi F, Guercio E, Maffiolini M, Magatti F, Spreafico L: Tension-free vaginal tape. Analysis of outcomes and complications in 404 stress incontinent women. Int Urogynecol J Suppl 2001;2:24-27.

-6 Schraffordt Koops SE, Bisseling TM, Heintz AP, Vervest HA: Prospective analysis of complications of tension-free vaginal tape from The Netherlands Tension-Free Vaginal Tape Study. Am J Obstet Gynecol 2005;193: 45-52.

7 Sokol IS, Jelovsek JE, Walters MD, Paraiso MFR, Barber MD: Incidence and predictors of prolonged urinary retention after TVT with and without concurrent prolapse surgery. Am J Obstet Gynecol 2005;192:15371543.

-8 Long CY, Lo TS, Liu CM, Hsu SC, Chang Y, Tsai EM: Lateral excision of tension-free vaginal tape for the treatment of iatrogenic urethral obstruction. Obstet Gynecol 2004;104: 1270-1274.

-9 Karram MM, Segal JL, Vassallo BJ, Kleeman SD: Complications and untoward effects of the tension-free vaginal tape procedure. Obstet Gynecol 2003;101:929-932.

-10 Klutke C, Siegel S, Carlin B, Paszkiewicz E, Kirkemo A, Klutke J: Urinary retention after tension-free vaginal tape procedure: incidence and treatment. Urology 2001;58:697701.
11 Nguyen JN: Tape mobilization for urinary retention after tension-free vaginal tape procedures. Urology 2005;66:523-526.

12 Rardin CR, Rosenblatt PL, Kohli N, Miklos JR, Heit M, Lucente VR: Release of tensionfree vaginal tape for the treatment of refractory postoperative voiding dysfunction. Obstet Gynecol 2002;100:898-902.

13 Doo CK, Hong B, Chung BJ, Kim JY, Jung HC, Lee KS, Choo MS: Five-year outcomes of tension-free vaginal tape procedure for treatment of female stress urinary incontinence. Eur Urol 2006;50:333-338.

14 Glavind K, Glavind E: Treatment of prolonged voiding dysfunction after tensionfree vaginal tape procedure. Acta Obstet Gynecol Scand 2007;86:357-360.

15 Ingelman-Sundberg A, Ulmsten U: Surgical treatment of female urinary stress incontinence; in Ulmsten U (ed): Female Stress Incontinence. Contrib Gynecol Obstet 1983; 10:51-69.

16 Wang KH, Neimark M, Davila GW: Voiding dysfunction following TVT procedure. Int Urogynecol J 2002;13:353-357.

17 Huang KH, Kung FT, Liang HM, Huang LY, Chang SY: Concomitant surgery with tension-free vaginal tape. Acta Obstet Gynecol Scand 2003;82:948-953.

18 Mishra VC, Mishra N, Karim OM, Motiwala HG: Voiding dysfunction after tension-free vaginal tape: a conservative approach is often successful. Int Urogynecol J 2005; 16: 210-214.

19 Mazouni C, Karsenty G, Bretelle F, Bladou F, Gamerre M, Serment G: Urinary complications and sexual function after the tensionfree vaginal tape procedure. Acta Obstet Gynecol Scand 2004;83:955-961.

20 Salin A, Conquy S, Elie C, Touboul C, Parra J, Zerbib M, Debré B, Amsellem-Ouazana D: Identification of risk factors for voiding dysfunction following TVT placement. Eur Urol 2007;51:782-787.

21 Dawson T, Lawton V, Adams E, Richmond D: Factors predictive of post-TVT voiding dysfunction. Int Urogynecol J Pelvic Floor Dysfunct 2007;18:1297-1302.
22 Pugsley H, Barbrook C, Mayne CJ, Tincello DG: Morbidity of incontinence surgery in women over 70 years old: a retrospective cohort study. BJOG 2005;112:786-790.

23 Duckett JR, Patil A, Papanikolaou NS: Predicting early voiding dysfunction after tension-free vaginal tape. J Obstet Gynaecol 2008;28:89-92.

24 Wang AC, Chen MC: The correlation between preoperative voiding mechanism and surgical outcome of the tension-free vaginal tape procedure with reference to quality of life. BJU Int 2003;91:502-506.

25 Nakata M: TVT multi-center study group: Voiding difficulty following tension-free vaginal tape procedure. Int Urogynecol J 2000;11:32-35.

-26 Bodelsson G, Henriksson L, Osser S, Stjernquist M: Short-term complications of the tension-free vaginal tape for stress urinary incontinence in women. $\mathrm{Br} J$ Obstet Gynaecol 2002;109:566-569.

27 Petri E, Niemeyer R, Martan A, Tunn R, Naumann G, Koelbl H: Reasons for and treatment of surgical complications with alloplastic slings. Int Urogynecol J 2006;17:313.

28 Partoll LM: Efficacy of tension-free vaginal tape with other pelvic reconstructive surgery. Am J Obstet Gynecol 2002;186:12921298.

29 Dietz HP, Ellis G, Wilson PD, Herbison P: Voiding function after tension-free vaginal tape. A longitudinal study. Aust N Z J Obstet Gynecol 2004;44:152-155.

30 Sander P, Sørensen F, Lose G: Does the tension-free vaginal tape procedure (TVT) affect the voiding function over time? Pressure-flow studies 1 year and $3 \frac{1}{2}$ years after TVT. Neurourol Urodyn 2007;26:995-997.

31 Liapis A, Bakas P, Creatsas G: Burch colposuspension and tension-free vaginal tape in the management of genuine stress incontinence in women. Eur Urol 2002;41:469-473.

32 Flock F, Kohorst F, Kreienberg R, Reich A: Ultrasound assessment of the tension-free vaginal tape (TVT). Ultraschall Med 2011; 32(suppl 1):S35-S40. 